\title{
Intra-retinal Layer Segmentation in Optical Coherence Tomography Using an Active Contour Approach
}

\author{
Azadeh Yazdanpanah ${ }^{1}$, Ghassan Hamarneh ${ }^{2}$, Benjamin Smith ${ }^{2}$, and Marinko Sarunic ${ }^{1}$ \\ 1 School of Engineering Science, \\ 2 Medical Image Analysis Lab, School of Computing Science, \\ Simon Fraser University, Canada \\ $\{$ aya18, hamarneh, brsmith, msarunic\}asfu.ca
}

\begin{abstract}
Optical coherence tomography (OCT) is a non-invasive, depth resolved imaging modality that has become a prominent ophthalmic diagnostic technique. We present an automatic segmentation algorithm to detect intra-retinal layers in OCT images acquired from rodent models of retinal degeneration. We adapt Chan-Vese's energy-minimizing active contours without edges for OCT images, which suffer from low contrast and are highly corrupted by noise. We adopt a multi-phase framework with a circular shape prior in order to model the boundaries of retinal layers and estimate the shape parameters using least squares. We use a contextual scheme to balance the weight of different terms in the energy functional. The results from various synthetic experiments and segmentation results on 20 OCT images from four rats are presented, demonstrating the strength of our method to detect the desired retinal layers with sufficient accuracy and average Dice similarity coefficient of 0.85 , specifically 0.94 for the the ganglion cell layer, which is the relevant layer for glaucoma diagnosis.
\end{abstract}

\section{Introduction}

Optical coherence tomography (OCT) is a novel non-invasive imaging modality which provides depth resolved structural information of a sample. The resolution in OCT systems approaches that of histology; the lateral resolution is typically $10-20 \mu \mathrm{m}$ and the axial resolution is typically $\sim 4 \mu \mathrm{m}[1]$.

OCT is a powerful tool for ophthalmic imaging and can be used to visualize the retinal cell layers to detect and monitor a variety of retinal diseases, including degeneration (thinning) of the retinal nerve cells layers due to glaucoma. OCT can be also adapted for imaging rodent eyes in order to complement medical research and gene therapy to combat retinal degeneration [2]. Fig. 1] shows a schematic of a rat's eye and a typical OCT depth profile of rodent retinal cell layers acquired in vivo. In this work, we developed an algorithm to automatically delineate the six retinal layers indicated in the figure in order to track glaucomatous degeneration.

Manual OCT segmentation is tedious, time-consuming, and suffers from inter- and intra-rater variability. Automated segmentation, on the other hand, holds the potential to reduce the time and effort required to delineate the retinal layers and also to provide repeatable, quantitative results. Several automated approaches have been employed in OCT segmentation [4]5]. They mostly rely on pixel-level, edge detection algorithms 


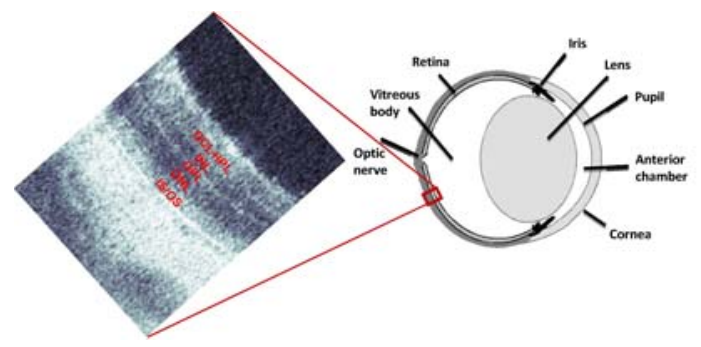

Fig. 1. A schematic anatomy of a rat's eye (right) is shown along with a typical OCT image (left) presenting a horizontal retinal cross-section with the labels indicating the retinal layers. The red box on the eye ball corresponds to the region which the image is taken from. The retina is divided into the inner and outer retina (IR and OR respectively). IR consists of the retinal nerve fiber layer, the inner ganglion cell layer (GCL), the inner plexiform layer (IPL), and the inner nuclear layer (INL). OR encompasses the outer plexiform layer (OPL), the outer nuclear layer (ONL), and the inner (IS) and outer (OS) segment photoreceptor layers [3].

such as the Canny-edge detector. Since OCT images are highly corrupted by speckle noise, some pre-processing steps are usually performed to reduce the effect of noise. The de-noising procedure, however, affects the sharpness of the edges which subsequently reduces the segmentation performance. Also, the conventional algorithms in OCT segmentation do not consider the intensity inhomogeneity in the image which can lead to inaccurate segments and inability to detect all layers. The predominant source of this artifact is the blood vessels on the topmost retinal layer which absorb light strongly, reducing the backscattered optical intensity of the underlying retinal tissue.

In this work, we propose a new method based on Chan-Vese active contour without edges [6] to address the segmentation of intra-retinal layers in OCT images. To the best of our knowledge, we are the first to segment OCT data using a multi-phase, level-set Mumford-Shah model that incorporates a shape prior based on expert anatomical knowledge of the retinal layers, avoiding the need for training. Our approach has four main features. First, it can segment all intra-retinal layers due to the multi-phase property of the algorithm. Second, we incorporate a shape prior term that enables the algorithm to accurately segment retinal layers, even where the region-based information is missing, such as in inhomogeneous regions. Third, our method is region-based and performs well on noisy OCT images. Finally, our algorithm is robust and avoids the re-initialization problem that is associated with the level set approach.

To achieve the needed accuracy and robustness for our application, we employ temporally and spatially adaptive (i.e. contextual) weights. We include concentric circles as a shape prior which mimic the true retinal layer structures and estimate the shape parameter using least squares. The methodology of our work is described in detail in Section 2 We apply the algorithm to 20 retinal OCT images acquired for both eyes of four rats. To evaluate the segmentation method, in Section 3, we measure the similarity between the automated segmentation with the ground truth manual segmentation using the Dice similarity coefficient (DSC). We conclude this paper in Section 4, with directions for future work to further automate our approach. 


\section{Methods}

Our objective is to segment a given OCT image, $I: \Omega \longrightarrow \mathbb{R}$, defined on the image domain, into $R$ disjoint sub-regions which accurately label the retinal layers. The decomposition of the image $I$, will be modeled using the level set framework as a set of $R-1$ signed distance functions (SDFs), $\phi$. Further details of this representation are provided in Section 2.1. To determine a segmentation, we developed an automatic, variational algorithm, that minimizes the following specific energy functional:

$$
E(\phi)=\lambda_{I} E_{I}(\phi)+\lambda_{S} E_{S}(\phi)+\lambda_{R} E_{R}(\phi)
$$

Each term of the energy functional captures a separate aspect of the problem. $E_{I}$, incorporates region-based information derived from the image. $E_{S}$ incorporates the prior shape knowledge of the anatomy of retinal layers. $E_{R}$ is a regularizing term which keeps region boundaries smooth and encourages each $\phi$ to be a SDF. Positive valued parameters, $\lambda_{I}, \lambda_{S}$, and $\lambda_{R}$, weight the different terms.

\subsection{Region-Based Energy Term}

The first term of equation (10 follows the work of Chan et al. [6] and encourages each region of the segmentation to have an approximately constant intensity. The intensity of the $i^{\text {th }}$ sub-region will be approximated by the constant $\mu_{i}$, and the spatial extent will be represented by a characteristic function $\chi_{i}$.

$$
E_{I}=\sum_{i=1}^{R} \int_{\Omega}\left(I-\mu_{i}\right)^{2} \chi_{i} d x d y
$$

By definition, each characteristic function takes the value 1 inside the region, and 0 outside. Following the approach of Mansouri et al. [7], each $\chi_{i}$ is represented using the level set method as a function of $R-1$ SDFs, $\phi$. Simply, $\chi_{i}$ is the region inside the zero contour (zero level set) of the $i^{t h} \mathrm{SDF}$, and outside all previous SDFs. The final region, $\chi_{R}$ is the region outside the zero contours of all SDFs. Using this partitioning, we guarantee unambiguous segmentation of $R$ regions using $(R-1)$ SDFs. The characteristic function for the $i^{t h}$ region is defined as follows, using the Heaviside step function, $H$, and delta function, $\delta[8]$ :

$$
\chi_{i}=H\left(\phi_{i}\right)^{1-\delta(R-i)}\left[\prod_{k=1}^{i-1}\left(1-H\left(\phi_{k}\right)\right)\right]
$$

\subsection{Shape Prior Energy Term}

OCT images may not always be piecewise constant. Intensity inhomogeneity may exist in regions due to the "shadows" of blood vessels on the topmost retinal layer. To compensate for these intensity inhomogeneities, we incorporated a shape prior term. Based on prior knowledge of retinal anatomy, a circular shape prior is used to model the retinal layer boundaries, and assists the algorithm when region-based information is insufficient to segment a layer accurately. In our model, each circular prior will share a 
common center point, but has a unique radius. The squared distance from a point, $(x, y)$, to the shape prior constraining the $i^{\text {th }}$ boundary, can be defined as:

$$
D_{i}(x, y)=\left[\left(x-c_{x}\right)^{2}+\left(y-c_{y}\right)^{2}-r_{i}^{2}\right]^{2}
$$

where $\left(c_{x}, c_{y}\right)$ is the common center of the concentric layers, and $r_{i}$ is the radius of the circular prior of the interface between the $i^{\text {th }}$ and the $(i+1)^{\text {th }}$ layer.

For each SDF $\phi_{i}$, a shape constraint encourages the region boundary (the zero contour) to lie on a circle, minimizing the squared distance of the zero contour to the prior. Consequently, the shape term in the energy functional is:

$$
E_{S}=\sum_{i=1}^{R-1} \int_{\Omega} D_{i}(x, y) \delta\left(\phi_{i}(x, y)\right)\left|\nabla \phi_{i}(x, y)\right| d x d y
$$

The term, $\delta\left(\phi_{i}(x, y)\right)\left|\nabla \phi_{i}(x, y)\right|$, selects out the zero contour of $\phi_{i}$. This causes the shape term to have a non-zero value only on the region boundaries, and the term $E_{S}$ is minimized when $\phi_{i}$ lies exactly on the circular shape.

The shape parameters, $c_{x}, c_{y}$ and $r_{i}$ for $E_{S}$ are defined using a least square fit, with

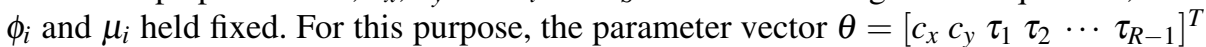
(where $\tau_{i}=r_{i}^{2}-c_{x}^{2}-c_{y}^{2}$ ) is estimated such that the error $\varepsilon$ in $b=\Psi \theta+\varepsilon$ is minimized, where $b$ and $\Psi$ are determined by points $(x, y)$ lying on $R-1$ boundaries.

\subsection{Regularization Energy Term}

Regularization terms were added to keep the boundary of the segmented layers smooth [6], and $\phi_{i}$ as a SDF. Smooth boundaries are encouraged by adding a contour length term, and $\phi_{i}$ can be kept close to a SDF by adding the penalty term of Chunming et al. [9]:

$$
E_{R}=\sum_{i=1}^{R-1} \int_{\Omega} \delta\left(\phi_{i}(x, y)\right)\left|\nabla \phi_{i}(x, y)\right|+\frac{1}{2}\left(\left|\nabla \phi_{i}(x, y)\right|-1\right)^{2} d x d y
$$

\subsection{Minimization of the Energy Functional}

By substituting the energy terms defined by (2), (5), and (6) in (1), and re-arranging slightly, the minimization problem associated with our model is defined as:

$$
\inf _{\mu_{i}, \phi_{i}} E=\int_{\Omega}\left\{\lambda_{I} \sum_{i=1}^{R}\left[\left(I-\mu_{i}\right)^{2} \chi_{i}\right]+\sum_{i=1}^{R-1}\left[A_{i}(x, y) \delta\left(\phi_{i}\right)\left|\nabla \phi_{i}\right|+\frac{1}{2}\left(\left|\nabla \phi_{i}\right|-1\right)^{2}\right]\right\} d x d y
$$

where $A_{i}(x, y)=\lambda_{R}+\lambda_{S} D_{i}(x, y)$.

To minimize this function, we followed the approach of Chan et al. [6] and performed an alternating minimization. First, we hold the SDFs fixed, and solve for the unknown intensities $\mu_{i}$ :

$$
\mu_{i}=\frac{\int_{\Omega} I(x, y) \chi_{i} d x d y}{\int_{\Omega} \chi_{i} d x d y}
$$


Next, holding the intensities fixed, we use the Euler-Lagrange equation with respect to $\phi_{i}$, and parameterize the descent direction using an artificial time $t$ :

$$
\frac{\partial \phi_{j}}{\partial t}=-\lambda_{I} \sum_{i=1}^{R}\left(I-\mu_{i}\right)^{2} \frac{\partial \chi_{i}}{\partial \phi_{j}}+\left[\nabla A_{j} \cdot \frac{\nabla \phi_{j}}{\left|\nabla \phi_{j}\right|}+A_{j} \operatorname{div}\left(\frac{\nabla \phi_{j}}{\left|\nabla \phi_{j}\right|}\right)\right] \delta\left(\phi_{j}\right)+\triangle \phi_{j}-\operatorname{div}\left(\frac{\nabla \phi_{j}}{\left|\nabla \phi_{j}\right|}\right)
$$

where

$$
\frac{\partial \chi_{i}}{\partial \phi_{j}}= \begin{cases}\left(-H\left(\phi_{i}\right)\right)^{1-\delta(i-j)} \delta\left(\phi_{j}\right) \prod_{k=1}^{i-1}\left(1-H\left(\phi_{k}\right)\right)^{1-\delta(k-j)} & i \neq R, j \leq i \\ -\delta\left(\phi_{j}\right) \prod_{k=1}^{i-1}\left(1-H\left(\phi_{k}\right)\right)^{1-\delta(k-j)} & i=R, j \leq i \\ 0 & i \neq R, \forall j>i\end{cases}
$$

Note that in practice, we must use regularized versions of $H$ and $\delta$ to obtain a welldefined descent direction. The regularization of Chan et. al [6] was used:

$$
H_{\varepsilon}(z)=\frac{1}{2}\left(1+\frac{2}{\pi} \arctan \left(\frac{z}{\varepsilon}\right)\right), \delta_{\varepsilon}(z)=\frac{1}{\pi} \frac{\varepsilon}{\varepsilon^{2}+z^{2}}
$$

\subsection{An Adaptive Weighting of Energy Terms}

Choosing "good" weights for energy terms in segmentation is an open problem, and finding the correct tradeoff that results in a desirable segmentation is usually treated empirically. In this work, we automatically adapt the weights both temporally and spatially, i.e. the weights change with iteration number and along the spatial dimensions. Intuitively, in early iterations, the region-based term should be more dominant, allowing the curve freedom to evolve toward the boundary of each layer. As the algorithm progresses, the shape term becomes more important to assist the algorithm when image information is insufficient to segment the image. Therefore, we define $\lambda_{I}$ and $\lambda_{S}$ in terms of the $n^{\text {th }}$ iteration as mentioned in [10]:

$$
\lambda_{I}(n)=\lambda_{I}(1)-\frac{n\left(\lambda_{I}(1)-\lambda_{I}(N)\right)}{N}, \quad \lambda_{S}(n)=\lambda_{S}(1)+\frac{\lambda_{S}(N)-\lambda_{S}(1)}{\cosh \left[8\left(\frac{n \pi}{N}-1\right)\right]}
$$

where $N$ is the total number of iterations.

We also want the shape term to have a greater effect where intensity information is missing, as in the inhomogeneous regions. Therefore, contextual information must be utilized. By choosing the weight of the shape term proportional to the inverse of the image gradient magnitude, we employ a spatially adaptive $\lambda_{S}$ in each iteration. As a result, the shape term has a higher weight than region-based term, for pixels on weak edges. This also has the beneficial effect that image pixels with higher gradient (strong edges) have a stronger influence when solving for shape prior parameters. More plainly, the least squares fitting of the shape prior parameters is weighted by image gradient.

\section{Results}

\subsection{Data Acquisition}

Images used in this study were acquired using a custom spectrometer based Fourier domain (FD)OCT system. The FDOCT system operated at a central wavelength of $826 \mathrm{~nm}$ 
and had an axial resolution of $\sim 4 \mu \mathrm{m}$ (in air). Non-invasive OCT imaging was performed on four wistar strain albino rats. One eye on each rat underwent an axotomy procedure (severing the optic nerve), the other eye was maintained as a control in order to monitor retinal degeneration. The axotomy procedure is an accelerated model of glaucoma and causes the retinal nerve fiber layer to thin as the ganglion cells die. Each eye was imaged four times over a period of two weeks using OCT. All animal imaging procedures were performed under protocols compliant to the Canadian Council on Animal Care with the approval of the University Animal Care Committee at SFU.

\subsection{Validation}

To qualitatively evaluate the performance of our approach, we compared the segmentation resulting from our method and from two other approaches, using the ground truth manual expert delineations on 20 OCT images. We refer to our method as the active contour without edge with shape constraint and contextual weights (ACWOE-SW). The two other approaches are the classical Chan-Vese's active contour (ACWOE) and the ACWOE with shape constraint only (ACWOE-S). For each method, the parameters were chosen to give the best results. Based on our experience, the initial and final values for $\lambda_{I}$ and $\lambda_{S}$ were set as follows: $\lambda_{I}(1)=1, \lambda_{I}(N)=0.5, \lambda_{S}(1)=0$, and $\lambda_{S}(N)=1$. $\lambda_{R}$ was set to $0.1 \times 255^{2}$. For all layers, the initial curve was estimated based on three points selected close to the interface of each layer. The same initialization was used for the three methods in all our experiments. Maximum of $N=100$ iterations were used which guaranteed convergence in all our experiments. Fig. 2 shows an example of the segmented results (red contours) for each method for a typical OCT retinal image along with the expert ground truth segmentation. As shown, ACWOE-SW detects all 6 interfaces between the retinal layer properly, revealing the performance of this model on the images with intensity inhomogeneity. Even very thin layers, such as INL and OPL, which are difficult to distinguish by eye, are segmented by the algorithm. In contrast, ACWOE failed to segment the IPL, INL, and OPL layers due to the intensity inhomogeneity and low contrast of the image. ACWOE-S shows better segmentation than ACWOE, but it still has poor performance in inhomogeneous regions.

To provide a quantitative evaluation of our approach, we measured the area similarity between the manual and automated segmentation using DSC $\in[0,1]$; more accurate segmentations correspond to higher DSC values. The average and standard deviation of DSC for the different retinal layers for all images is summarized for our method

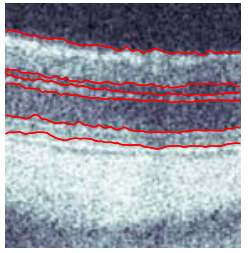

(a) ACWOE-SW

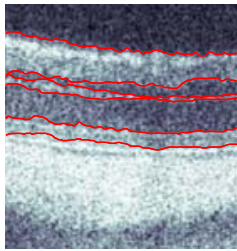

(b) ACWOE-S

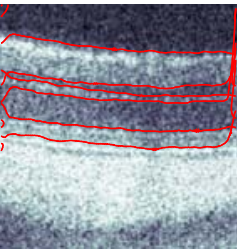

(c) ACWOE

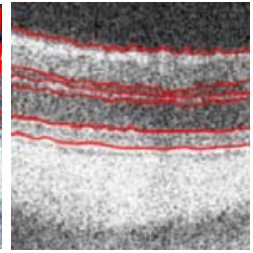

(d) Manual

Fig. 2. Segmentation results for an OCT retinal image 


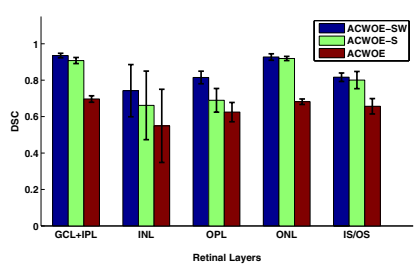

(a)

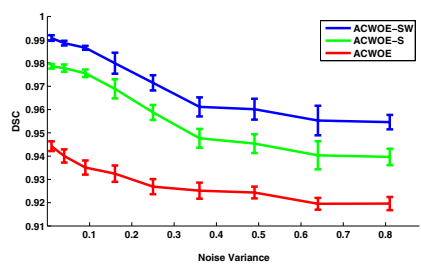

(b)

Fig. 3. (a) Segmentation results for different retinal layers. (b) DSC for different noise levels in the synthetic data; the image intensity is normalized to range from 0 to 1 .

(ACWOE-SW) as: GCL+IPL (0.94 \pm 0.012$)$, INL (0.74 \pm 0.143$)$, OPL (0.82 \pm 0.034$)$, ONL $(0.93 \pm 0.018)$, and IS/OS $(0.82 \pm 0.023)$. Fig. 3(a) provides a quantitative comparison between the DSC of ACWOE-SW for the 20 images versus ACWOE-S, and ACWOE for different retinal layers. Our method is superior to the other approaches for all the examined layers.

\subsection{Noise Tolerance}

In order to evaluate the robustness of our algorithm to noise, controlled synthetic retinal OCT-like images were created with a known ground truth segmentation but corrupted with varying degrees of additive white Gaussian noise. As shown in Fig. 3(b) the DSC performance results reveal that adding a shape constraint to the energy functional makes the algorithm more accurate than standard ACWOE, while remaining robust in the presence of noise.

\section{Discussion and Conclusion}

We developed an automatic iterative algorithm to segment low contrast OCT images of rodent retinal layers. We used a multi-phase framework with a circular shape prior. We have demonstrated that our approach is able to accurately segment all of the intraretinal layers, even given their small size and similar texture. Our approach also shows an improved performance in regions with intensity inhomogeneity due to the inclusion of shape prior constraint. We also introduced a contextual scheme to balance the weight of different terms in the energy functional which makes the algorithm even more robust when the image information is not sufficient to accurately detect layers. In addition, ACWOE-SW is more accurate in comparison with other active contours in the presence of noise. To demonstrate the robustness and performance of the algorithm, we applied it to both synthetic data and retina images from rats. The experimental results showed that we could classify the desired retinal layers. However, due to the various applications of OCT in diagnosis of retina diseases, we need a fully automatic segmentation algorithm which is more acceptable in clinical applications. Therefore, in future work, we will make the algorithm independent of the user by eliminating the dependency on the position of the initial curve by adopting convex functionals. We also need to apply our algorithm on a larger database from rats and assess the sensitivity of the algorithm 
with respect to the parameters and initial curve. Finally, we plan to expand our algorithm to human OCT images. Migrating our ACWOE-SW method to segment human retina images will require changing the shape prior to incorporate the foveal pit, but otherwise is anticipated to provide similarly high accuracy segmentation results.

Acknowledgments. The authors would like to thank Dr. H. Saragovi at McGill University for providing the axotomy rats. We also acknowledge support from MSFHR Career Investigator Award to MVS and grant support from NSERC/CIHR CHRP (MVS) and $\operatorname{NSERC}(\mathrm{GH})$.

\section{References}

1. Fercher, A.F., Drexler, W., Hitzenberger, C.K., Lasser, T.: Optical coherence tomographyprinciples and applications. Reports on progress in physics 66, 239-303 (2003)

2. Walsh, A., Sadda, S.R.: Optical coherence tomography in the diagnosis of retinal vascular disease. In: Retinal Vascular Disease, pp. 205-227. Springer, Heidelberg (2007)

3. Baroni, M., Diciotti, S., Evangelisti, A., Fortunato, P., Torre, A.L.: Texture classification of retinal layers in optical coherence tomography. In: 11th Mediterranean Conference on Medical and Biomedical Engineering and Computing, pp. 847-850 (2007)

4. Fernández, D.C., Salinas, H.M., Puliafito, C.A.: Automated detection of retinal layer structures on optical coherence tomography images. Opt. Express 13(25), 10200-10216 (2005)

5. Bagci, A.M., Shahidi, M., Ansari, R., Blair, M., Blair, N.P., Zelkha, R.: Thickness profiles of retinal layers by optical coherence tomography image segmentation. American Journal of Ophthalmology 146(5), 679-687 (2008)

6. Chan, T., Vese, L.: Active contours without edges. IEEE Trans. on Image Processing 10(2), 266-277 (2001)

7. Mansouri, A.R., Mitiche, A., Vázquez, C.: Multiregion competition: A level set extension of region competition to multiple region image partitioning. Computer Vision and Image Understanding 101(3), 137-150 (2006)

8. Smith, B., Saad, A., Hamarneh, G., Moeller, T.: Recovery of dynamic PET regions via simultaenous segmentation and deconvolution. In: MICCAI Workshop on Analysis of Functional Medical Image Data (MICCAI functional), pp. 33-40 (2008)

9. Li, C., Xu, C., Gui, C., Fox, M.: Level set evolution without re-initialization: a new variational formulation. In: IEEE Computer Society Conference on Computer Vision and Pattern Recognition (CVPR), pp. 430-436 (2005)

10. Pluempitiwiriyawej, C., Moura, J., Wu, Y.J.L., Ho, C.: STACS: new active contour scheme for cardiac MR image segmentation. IEEE Trans. on Medical Imaging 24(5), 593-603 (2005) 